\title{
Exponential stability of perturbed linear discrete systems
}

\author{
Josef Diblík ${ }^{1 *}$, Denys Y Khusainov ${ }^{2}$, Jaromír Baštinec ${ }^{1}$ and Andrii S Sirenko ${ }^{2}$
}

${ }^{\text {*Correspondence: }}$ diblik.j@fce.vutbr.cz

${ }^{1}$ Brno University of Technology, Brno, Czech Republic

Full list of author information is

available at the end of the article

\begin{abstract}
The paper considers the problem of exponential stability and convergence rate to solutions of perturbed linear discrete homogeneous systems. New criteria on exponential stability are derived by using the second method of Lyapunov. We consider non-delayed systems as well as systems with a single delay. Simultaneously, explicit exponential estimates of the solutions are derived. The results are illustrated by examples.
\end{abstract}

\section{Introduction}

The dynamics of discontinuous transition states of dynamic systems is most naturally described by difference equations and investigating the properties of difference equations is a very important area of research (we mention at least the monographs [1-6]). If processes are modeled by systems of equations, in general, some of the model parameters may be uncertain and we have to deal with systems with inaccurately specified parameters.

In the paper, we consider the exponential stability of systems of perturbed linear homogeneous difference equations and give explicit exponential estimates of solutions. In such systems, it is usually appropriate to describe the inaccurately specified parameters as perturbations of some initial systems. Investigated are non-delayed homogeneous systems of difference equations and homogeneous systems of difference equations with a single delay.

Let $\mathcal{A}$ be a symmetric positive definite matrix. Denote by $\lambda_{\max }(\mathcal{A}), \lambda_{\min }(\mathcal{A})$ its maximum and minimum eigenvalues and let

$$
\varphi(\mathcal{A}):=\frac{\lambda_{\max }(\mathcal{A})}{\lambda_{\min }(\mathcal{A})} .
$$

Throughout the paper, for an arbitrary matrix $\mathcal{B}$, we use the matrix norm

$$
|\mathcal{B}|=\sqrt{\lambda_{\max }\left(\mathcal{B}^{T} \mathcal{B}\right)}
$$

This norm reduces to the Euclidean norm

$$
\begin{gathered}
|x|=\sqrt{\sum_{i=1}^{n} x_{i}^{2}} \\
\text { if } \mathcal{B}=x=\left(x_{1}, \ldots, x_{n}\right)^{T} .
\end{gathered}
$$

(c) 2016 Diblík et al. This article is distributed under the terms of the Creative Commons Attribution 4.0 International License (http://creativecommons.org/licenses/by/4.0/), which permits unrestricted use, distribution, and reproduction in any medium, provided you give appropriate credit to the original author(s) and the source, provide a link to the Creative Commons license, and indicate if changes were made. 
Now we describe the problems solved in the paper and the methods frequently used to investigate stability.

\subsection{Non-delayed systems}

The simplest class of systems of difference equations is that of linear autonomous systems

$$
x(k+1)=A x(k), \quad k=0,1, \ldots,
$$

where $A=\left(a_{i j}\right)_{i, j=1}^{n}$ is a real constant matrix and $x$ is an $n$-dimensional unknown vector. In the paper, we consider systems

$$
x(k+1)=(A+B(k)) x(k), \quad k=0,1, \ldots,
$$

where the norm $|B(k)|$ of the real matrix $B(k)=\left(b_{i j}(k)\right)_{i, j=1}^{n}$ is small in a certain sense specified below. System (2) can be viewed as a perturbation of system (1). Recall that the solution of (1) or (2) is uniquely determined by the given initial value $x(0)$.

Definition 1 The zero solution $x(k)=0, k=0,1, \ldots$ of (2) is called (Lyapunov) stable if, for an arbitrary $\varepsilon>0$, there exists a $\delta(\varepsilon)>0$ such that, for any solution $x^{*}(k)$ of system (2), we have $\left|x^{*}(k)\right|<\varepsilon$ for $k=0,1, \ldots$, provided that $\left|x^{*}(0)\right|<\delta(\varepsilon)$. If, moreover,

$$
\lim _{k \rightarrow+\infty}\left|x^{*}(k)\right|=0
$$

the zero solution is called asymptotically stable. The zero solution of (2) is called exponentially stable if there exist constants $N>0$ and $\theta \in(0,1)$ not depending on $x^{*}$ such that

$$
\left|x^{*}(k)\right| \leq N\left|x^{*}(0)\right| \theta^{k}, \quad k=1,2, \ldots
$$

For the basic notions, properties, and results we refer, e.g., to $[1,4,6,7]$ and to the references therein. For linear systems, all solutions are simultaneously stable (exponentially stable) if the zero solution is stable (exponentially stable). Therefore, in contrast to nonlinear systems, for linear systems, the concept of a stable system (exponentially stable system) is meaningful.

The stability of system (1) is often analyzed through the characteristic equation

$$
\operatorname{det}(\lambda I-A)=0
$$

where $I$ is an $n \times n$ identity matrix and $\lambda$ is a suitable complex constant. Expanding (4), we obtain

$$
\lambda^{n}+p_{1} \lambda^{n-1}+p_{2} \lambda^{n-2}+\cdots+p_{n-1} \lambda+p_{n}=0
$$

with real $p_{i}, i=1,2, \ldots, n$. Necessary and sufficient for the exponential stability of the linear system (1) is the condition $\rho(A)<1$ (see, e.g., $[4,6]$ ) where $\rho(A)$ is the spectral radius of the matrix $A$. For discrete equations, unfortunately, such a simple and effective tool of investigation of the roots of the characteristic equation for the differential systems, as the 
Hurwitz criterion giving the necessary and sufficient condition for $\operatorname{Re} \lambda_{i}<0, i=1,2, \ldots, n$, is not available. The well-known Schur-Cohn criterion $[4,8,9]$, to verify $\rho(A)<1$, is not easily applicable because the higher the order of the characteristic equation (5), the more difficult the computation becomes.

An alternative to studying the stability by algebraic methods is the method of Lyapunov functions (described, e.g., in [4, 6]). For linear stationary systems (1), the Lyapunov function is sought in the form of a quadratic form $V(x)=x^{T} H x$ where a symmetric positive definite $n \times n$ matrix $H$ is the solution of the matrix Lyapunov equation

$$
A^{T} H A-H=-C \text {. }
$$

System (1) is exponentially stable if and only if, for an arbitrary positive definite symmetric $n \times n$ matrix $C$, the matrix equation (6) is solvable and has a unique solution - a positive definite symmetric matrix $H$ [4].

The parameters of mathematical models of dynamic systems are, generally speaking, determined experimentally and are known with some degree of adequacy. The stability of such systems can be investigated as the stability of a perturbed system to a given initial system.

The investigation of the stability of system (2) is, in essence, close to the investigation of what is called interval stability of linear differential equations. Such an investigation was carried out by Haritonov, we refer at least to one of his founding papers [10] where the necessary and sufficient conditions for stability were formulated.

\subsection{Systems with a single delay}

In Section 3, we study linear homogeneous difference systems with a single delay,

$$
x(k+1)=(A+B(k)) x(k)+(D+E(k)) x(k-m), \quad k=0,1, \ldots,
$$

where $A=\left(a_{i j}\right)_{i, j=1}^{n}$ and $D=\left(d_{i j}\right)_{i, j=1}^{n}$ are real constant matrices, $m \in \mathbb{N}$, and norms $|B(k)|$, $|E(k)|$ of the real matrices $B(k)=\left(b_{i j}(k)\right)_{i, j=1}^{n}, E(k)=\left(e_{i j}(k)\right)_{i, j=1}^{n}$ are small in a sense. System (7) can be viewed as a perturbation of the system

$$
x(k+1)=A x(k)+D x(k-m), \quad k=0,1, \ldots
$$

Recall that the initial problem $x(k)=x_{k}^{*}, k=-m,-m+1, \ldots, 0$ for (7) or (8) where $x_{k}^{*} \in \mathbb{R}^{n}$ is uniquely solvable. Below, we utilize the norm

$$
\|x(0)\|_{m}:=\max \{|x(i)|, i=-m,-m+1, \ldots, 0\} .
$$

Definition 2 The zero solution $x(k)=0, k=-m,-m+1, \ldots$ of system (7) is called (Lyapunov) stable if, for an arbitrary $\varepsilon>0$, there exists a $\delta(\varepsilon)>0$ such that, for any solution $x^{*}(k)$ of system (7), we have $\left|x^{*}(k)\right|<\varepsilon$ for $k=0,1, \ldots$, provided that $\left\|x^{*}(0)\right\|_{m}<\delta(\varepsilon)$. If, moreover,

$$
\lim _{k \rightarrow+\infty}\left|x^{*}(k)\right|=0,
$$


the zero solution is called asymptotically stable. The zero solution of system (7) is called exponentially stable if there exist constants $N>0$ and $\theta \in(0,1)$ not depending on $x^{*}$ such that

$$
\left|x^{*}(k)\right| \leq N\left\|x^{*}(0)\right\|_{m} \theta^{k}, \quad k=1,2, \ldots
$$

To establish the fact of the asymptotic stability of system (8), we consider the associated characteristic equation

$$
\operatorname{det}\left(\lambda^{m+1} I-\lambda^{m} A-D\right)=0 .
$$

Expanding the determinant on the right-hand side, we obtain, in general, an algebraic equation of degree $(m+1) n$ and we are facing an even greater difficulty than in the case of equation (5). Recently, for the asymptotic stability of special classes of linear discrete equations with constant complex coefficients and with delay, simple criterions have been derived in [11-17] (the criteria derived in [11] are fully explicit with respect to the delay).

The paper is organized as follows. Sufficient conditions for the exponential stability and explicit exponential estimates of solutions of non-delayed systems (2) are derived in Section 2 by the second method of Lyapunov. For systems with a single delay (7), such an investigation is performed in Section 3. The investigations of both classes of systems (2) and (7) differ. The difference is caused by the presence of delay in (7). The difficulty caused by the Lyapunov function being estimated was overcome by using what is called Razumikhin condition while applying of several auxiliary inequalities. The applicability of the results is illustrated by examples and concluding remarks are included in Section 4.

\section{Exponential stability of non-delayed systems}

Theorem 1 below gives sufficient conditions for exponential stability of the perturbed system (2), and suitable parameters $N$ and $\theta$ in the definition of exponential stability (3) of solutions are explicitly defined. In the proof, Lyapunov function $V(x)=x^{T} H x$ is used where $H$ solves the matrix Lyapunov equation (6). With respect to the matrix of perturbation $B(k), k=0,1, \ldots$ we assume that there exists a number $\mathcal{D}$ such that, for every $k=0,1, \ldots$, we have

$$
|B(k)| \leq \mathcal{D}
$$

First, we need auxiliary lemmas. Denote

$$
\mathcal{D}^{*}:=\frac{1}{\lambda_{\max }(H)}\left[\sqrt{\left|A^{T} H\right|^{2}+\lambda_{\min }(C) \lambda_{\max }(H)}-\left|A^{T} H\right|\right]
$$

and

$$
\xi:=\lambda_{\min }(C)-2\left|A^{T} H\right| \mathcal{D}-|H| \mathcal{D}^{2} .
$$

Lemma 1 Let $\rho(A)<1$. Let $C$ be a fixed positive definite matrix and let matrix $H$ solve matrix equation (6). Then $\mathcal{D}^{*}>0$ and, if $\mathcal{D}<\mathcal{D}^{*}$, then $\xi>0$. 
Proof Inequality $\mathcal{D}^{*}>0$ is obvious. Solve the quadratic equation $\xi=0$ with respect to $\mathcal{D}$. Its roots $\mathcal{D}_{1,2}$ are

$$
\mathcal{D}_{1,2}=\frac{1}{\lambda_{\max }(H)}\left[-\left|A^{T} H\right| \pm \sqrt{\left|A^{T} H\right|^{2}+\lambda_{\min }(C) \lambda_{\max }(H)}\right]
$$

and $\xi>0$ if $\mathcal{D} \in\left(\mathcal{D}_{2}, \mathcal{D}_{1}\right)=\left(\mathcal{D}_{2}, \mathcal{D}^{*}\right), \mathcal{D}_{2}<0$. Since, by its definition, $\mathcal{D} \geq 0$, we conclude that $\xi>0$ if $\mathcal{D} \in\left[0, \mathcal{D}^{*}\right)$.

The following result is taken from Chapter 7, Section 4 in [18].

Lemma 2 Let $A_{1}$ and $A_{2}$ be positive definite matrices. Then

$$
\lambda_{\min }\left(A_{1}+A_{2}\right) \geq \lambda_{\min }\left(A_{1}\right)+\lambda_{\min }\left(A_{2}\right) .
$$

In the Russian translation of [18], it is noted that (10) holds even for arbitrary Hermitian matrices $A_{1}, A_{2}$.

Theorem 1 Let $\rho(A)<1$. Let $C$ be a fixed positive definite matrix and let matrix $H$ solve the matrix Lyapunov equation (6). If

$$
\mathcal{D}<\mathcal{D}^{*},
$$

then system (2) is exponentially stable and, for any of its solutions $x=x(k)$, the inequality

$$
|x(k)| \leq\left[1-\frac{\xi}{\lambda_{\max }(H)}\right]^{k / 2} \sqrt{\varphi(H)}|x(0)|, \quad k \geq 0,
$$

holds where

$$
0<1-\frac{\xi}{\lambda_{\max }(H)}<1 .
$$

Proof Recall that $H$ is a symmetric positive definite matrix and, by Lemma $1, \xi>0$ since (11) is assumed. Define a Lyapunov function

$$
V(x):=x^{T} H x .
$$

For the first difference of $V(x)$ along the trajectories of system (2), we have $(k \geq 0)$

$$
\begin{aligned}
\Delta V(x(k)) & =V(x(k+1))-V(x(k)) \\
& =(A x(k)+B(k) x(k))^{T} H(A x(k)+B(k) x(k))-x^{T}(k) H x(k) .
\end{aligned}
$$

We transform the last difference to

$$
\begin{aligned}
\Delta V(x(k))= & x^{T}(k)\left(A^{T} H A-H\right) x(k)+2 x^{T}(k) A^{T} H B(k) x(k) \\
& +x^{T}(k)(B(k))^{T} H B(k) x(k)
\end{aligned}
$$




$$
\begin{aligned}
& \leq-\lambda_{\min }(C)|x(k)|^{2}+2\left|A^{T} H\right| \mathcal{D}|x(k)|^{2}+|H| \mathcal{D}^{2}|x(k)|^{2} \\
& =-\xi|x(k)|^{2} .
\end{aligned}
$$

Consequently, $\Delta V(x(k)) \leq 0$, and the zero equilibrium (and, therefore, system (2)) is stable. Now we derive an explicit estimate (12) of the exponential type. Since

$$
\lambda_{\min }(H)|x|^{2} \leq V(x) \leq \lambda_{\max }(H)|x|^{2},
$$

from inequality (14) we get

$$
V(x(k+1))-V(x(k)) \leq-\xi|x(k)|^{2} \leq-\frac{\xi}{\lambda_{\max }(H)} V(x(k))
$$

or

$$
V(x(k+1)) \leq\left[1-\frac{\xi}{\lambda_{\max }(H)}\right] V(x(k)) .
$$

Below we solve this difference inequality, but first we show that the coefficient

$$
1-\frac{\xi}{\lambda_{\max }(H)}
$$

on the right-hand side of (16) satisfies estimates (13). The right-hand side of inequality (13) is a simple consequence of inequality $\xi>0$.

To prove the left-hand side of inequality (13), we use Lemma 2 with matrices $A_{1}, A_{2}$ defined as $A_{1}:=H-A^{T} H A$ and $A_{2}:=A^{T} H A$. Then

$$
\begin{aligned}
\lambda_{\min }(H) & =\lambda_{\min }\left(\left(H-A^{T} H A\right)+A^{T} H A\right) \\
& =\lambda_{\min }\left(A_{1}+A_{2}\right) \geq \lambda_{\min }\left(A_{1}\right)+\lambda_{\min }\left(A_{2}\right) \\
& =\lambda_{\min }\left(H-A^{T} H A\right)+\lambda_{\min }\left(A^{T} H A\right)
\end{aligned}
$$

and

$$
\begin{aligned}
\lambda_{\min }(C) & =\lambda_{\min }\left(H-A^{T} H A\right) \leq \lambda_{\min }(H)-\lambda_{\min }\left(A^{T} H A\right) \\
& \leq \lambda_{\max }(H)-\lambda_{\min }\left(A^{T} H A\right)<\lambda_{\max }(H) .
\end{aligned}
$$

Therefore,

$$
\xi=\lambda_{\min }(C)-2\left|A^{T} H\right| \mathcal{D}-|H| \mathcal{D}^{2}<\lambda_{\max }(H)
$$

and the left-hand side of inequality (13) holds.

Solving the difference inequality (16), we obtain

$$
V(x(k)) \leq\left[1-\frac{\xi}{\lambda_{\max }(H)}\right]^{k} V(x(0)), \quad k \geq 0
$$


Using (15) together with the obtained inequality, we get

$$
\begin{aligned}
\lambda_{\min }(H)|x(k)|^{2} & \leq V(x(k)) \leq\left[1-\frac{\xi}{\lambda_{\max }(H)}\right]^{k} V(x(0)) \\
& \leq \lambda_{\max }(H)|x(0)|^{2}\left[1-\frac{\xi}{\lambda_{\max }(H)}\right]^{k}, \quad k \geq 0,
\end{aligned}
$$

and, consequently,

$$
|x(k)|^{2} \leq\left[1-\frac{\xi}{\lambda_{\max }(H)}\right]^{k} \frac{\lambda_{\max }(H)}{\lambda_{\min }(H)}|x(0)|^{2}=\left[1-\frac{\xi}{\lambda_{\max }(H)}\right]^{k} \varphi(H)|x(0)|^{2}, \quad k \geq 0
$$

or

$$
|x(k)| \leq\left[1-\frac{\xi}{\lambda_{\max }(H)}\right]^{k / 2} \sqrt{\varphi(H)}|x(0)|, \quad k \geq 0
$$

i.e., we get inequality (12).

Example 1 Let $n=2$ and

$$
A=\left(\begin{array}{ll}
0.7 & 0.1 \\
0.1 & 0.7
\end{array}\right)
$$

be specified in system (2). Let

$$
C=\left(\begin{array}{cc}
0.5 & -0.14 \\
-0.14 & 0.5
\end{array}\right)
$$

The matrix

$$
H=\left(\begin{array}{ll}
1 & 0 \\
0 & 1
\end{array}\right)
$$

solves the matrix Lyapunov equation (6), $\lambda_{\min }(C)=0.36, \lambda_{\min }(H)=\lambda_{\max }(H)=\varphi(H)=1$, $|H|=1,\left|A^{T} H\right|=0.8$ and $\mathcal{D}^{*}=0.2$. If $|B(k)| \leq \mathcal{D}<\mathcal{D}^{*}=0.2, k=0,1, \ldots$, then system (2) is exponentially stable. For, say, $\mathcal{D}=0.19$, we have $\xi=0.0199$ and (12) becomes

$$
|x(k)| \leq\left[1-\frac{\xi}{\lambda_{\max }(H)}\right]^{k / 2} \sqrt{\varphi(H)}|x(0)|=(0.9801)^{k / 2} \cdot|x(0)|, \quad k \geq 0
$$

\section{Exponential stability and estimation of convergence of linear systems with delay}

In this part we study linear difference systems with single delay (7) by the second Lyapunov method. A short survey of the use of the second Lyapunov method for the investigation of stability of discrete systems with delay now follows. Often, two modifications are used. The first is what is called the method of finite-dimensional Lyapunov functions, and the second one is the method of functionals of Lyapunov-Krasovskii. Strictly speaking, the 
latter method is called the method of functionals only because it is a discrete analog of the method of functionals for functional differential equations with delay. In the following, we use the former method.

If the method of Lyapunov functions is to be applied to systems of differential equations with delay $\dot{x}(t)=f(x(t), x(t-\tau))$ where $f: \mathbb{R}^{n} \times \mathbb{R}^{n} \rightarrow \mathbb{R}^{n}$ and $\tau>0$, it is necessary to find a positive definite and continuously differentiable function $V(x)$ such that its total derivative along solutions of the system is negative definite provided that a so-called Razumikhin condition holds, i.e., that the solutions approach the surface level

$$
\partial V^{\alpha}=\left\{x \in R^{n}: V(x)=\alpha\right\},
$$

where $\alpha$ is a positive constant from the domain

$$
V^{\alpha}=\left\{x \in R^{n}: V(x)<\alpha\right\} .
$$

Formally, the Razumikhin condition can be written as $V(x(s))<V(x(t))$ where $t-\tau \leq s<t$. For quadratic Lyapunov functions $V(x)=x^{T} H x$ with a symmetric positive definite matrix $H$, this inequality implies $|x(s)|<\sqrt{\varphi(H)}|x(t)|$ where $t-\tau \leq s<t$. Below, we consider a similar approach for studying the stability of difference systems with delay.

\subsection{Auxiliary inequalities}

First we derive three lemmas related to auxiliary inequalities. Such inequalities are used in the proof of Theorem 2 in Section 3.2.

Lemma 3 Let $L_{0}>0, a_{0} \geq 0, b_{0} \geq 0, c_{0}>0, d_{0} \geq 0$, and $e_{0}>0$ be constants. If there exist positive constants $\xi$ and $\eta$ such that

$$
|B(k)|<\xi, \quad|E(k)|<\eta, \quad k \geq 0,
$$

and $\xi<\xi_{0}, \eta<\eta_{0}$ where

$$
\begin{aligned}
\xi_{0} & =\frac{-a_{0}+\sqrt{a_{0}^{2}+2 c_{0} L_{0}}}{2 c_{0}}, \\
\eta_{0} & =\frac{-\left(b_{0}+d_{0} \xi_{0}\right)+\sqrt{\left(b_{0}+d_{0} \xi_{0}\right)^{2}+2 e_{0} L_{0}}}{2 e_{0}},
\end{aligned}
$$

then

$$
L_{0}-a_{0}|B(k)|-b_{0}|E(k)|-c_{0}|B(k)|^{2}-d_{0}|B(k)||E(k)|-e_{0}|E(k)|^{2}>0, \quad k \geq 0 .
$$

Proof If

$$
L_{0}-a_{0} \xi-b_{0} \eta-c_{0} \xi^{2}-d_{0} \xi \eta-e_{0} \eta^{2}>0,
$$

then (20) holds. Let $\xi$ be such that

$$
c_{0} \xi^{2}+a_{0} \xi-\frac{1}{2} L_{0}<0
$$


The above inequality holds if $0<\xi<\xi_{0}$ where $\xi_{0}$ is defined by (18). Now we find $\eta$ such that

$$
e_{0} \eta^{2}+\left(b_{0}+d_{0} \xi_{0}\right) \eta-\frac{1}{2} L_{0}<0
$$

The last inequality is valid if $0<\eta<\eta_{0}$ where $\eta_{0}$ is defined by (19). Therefore, (20) will be valid for $0<\xi<\xi_{0}, 0<\eta<\eta_{0}$ and $k \geq 0$.

Lemma 4 Let $L_{1}>0, a_{1} \geq 0, b_{1} \geq 0, c_{1}>0$, and $d_{1}>0$ be constants. If there exist positive constants $\xi$ and $\eta$ such that

$$
|B(k)|<\xi, \quad|E(k)|<\eta, \quad k \geq 0,
$$

and $\xi<\xi_{1}, \eta<\eta_{1}$ where

$$
\begin{aligned}
& \xi_{1}=\frac{-a_{1}+\sqrt{a_{1}^{2}+2 c_{1} L_{1}}}{2 c_{1}}, \\
& \eta_{1}=\frac{L_{1}}{2\left(b_{1}+d_{1} \xi_{1}\right)},
\end{aligned}
$$

then

$$
L_{1}-a_{1}|B(k)|-b_{1}|E(k)|-c_{1}|B(k)|^{2}-d_{1}|B(k)||E(k)|>0, \quad k \geq 0 .
$$

Proof If

$$
L_{1}-a_{1} \xi-b_{1} \eta-c_{1} \xi^{2}-d_{1} \xi \eta>0,
$$

then (24) holds. Assume that $\xi$ satisfies

$$
c_{1} \xi^{2}+a_{1} \xi-\frac{1}{2} L_{1}<0
$$

This inequality holds if $0<\xi<\xi_{1}$ where $\xi_{1}$ is defined by (22). Now we find $\eta$ satisfying

$$
\left(b_{1}+d_{1} \xi_{1}\right) \eta-\frac{1}{2} L_{1}<0
$$

This inequality is valid if $0<\eta<\eta_{1}$ where $\eta_{1}$ is defined by (23). Therefore, (24) will be valid for $0<\xi<\xi_{1}, 0<\eta<\eta_{1}$, and $k \geq 0$.

Lemma 5 Let $L_{2}>0, a_{2} \geq 0, b_{2} \geq 0, d_{2}>0$, and $e_{2}>0$ be constants. If there exist positive constants $\xi$ and $\eta$ such that

$$
|B(k)|<\xi, \quad|E(k)|<\eta, \quad k \geq 0,
$$

and $\xi<\xi_{2}, \eta<\eta_{2}$ where

$$
\eta_{2}=\frac{-b_{2}+\sqrt{b_{2}^{2}+2 e_{2} L_{2}}}{2 e_{2}},
$$




$$
\xi_{2}=\frac{L_{2}}{2\left(a_{2}+d_{2} \eta_{2}\right)},
$$

then

$$
L_{2}-a_{2}|B(k)|-b_{2}|E(k)|-d_{2}|B(k)||E(k)|-e_{2}|E(k)|^{2}>0, \quad k \geq 0 .
$$

Proof If

$$
L_{2}-a_{2} \xi-b_{2} \eta-d_{2} \xi \eta-e_{2} \eta^{2}>0,
$$

then (28) holds. We find $\eta$ such that

$$
e_{2} \eta^{2}+b_{2} \eta-\frac{1}{2} L_{2}<0 \text {. }
$$

The last inequality will hold if $0<\eta<\eta_{2}$ where $\eta_{2}$ is defined by (26). Now we choose $\xi$ such that inequality

$$
a_{2} \xi+d_{2} \eta_{2} \xi-\frac{1}{2} L_{2}<0
$$

holds, i.e., only inequality $0<\xi<\xi_{2}$ needs to be satisfied where $\xi_{2}$ is defined by (27). Therefore, (28) will be valid for $0<\xi<\xi_{2}, 0<\eta<\eta_{2}$, and $k \geq 0$.

\subsection{Exponential stability and estimation of solutions}

Let $\gamma>1$. To apply the auxiliary lemmas from the previous part in the proof of Theorem 2, the following quantities are needed:

$$
\begin{aligned}
& L_{0}:=\lambda_{\min }(C)-\left(1+\gamma \varphi^{2}(H)\right)\left|A^{T} H D\right|-\gamma \varphi^{2}(H)\left|D^{T} H D\right|-(1-1 / \gamma)|H|, \\
& a_{0}:=2|H A|+\left(1+\gamma \varphi^{2}(H)\right)|H D|, \quad b_{0}:=|H A|+[2|H D|+|H A|] \gamma \varphi^{2}(H), \\
& c_{0}:=|H|, \quad d_{0}:=\left(1+\gamma \varphi^{2}(H)\right)|H|, \quad e_{0}:=\gamma|H| \varphi^{2}(H), \\
& L_{11}:=\lambda_{\min }(C)-\left|A^{T} H D\right|-(1-1 / \gamma)|H|, \\
& a_{11}:=(2|H A|+|H D|), \quad b_{11}:=|H A|, \quad c_{11}=d_{11}:=|H|, \\
& L_{12}:=\lambda_{\max }(H)-\gamma\left[\lambda_{\min }(C)-\left|A^{T} H D\right|\right]-(\gamma-1)|H|, \\
& a_{12}:=\gamma(2|H A|+|H D|), \quad b_{12}:=\gamma|H A|, \quad c_{12}=d_{12}:=\gamma|H|, \\
& L_{2}:=\lambda_{\min }(H)-\gamma^{m+1}\left[\left|A^{T} H D\right|+\left|D^{T} H D\right|\right] \varphi(H), \\
& a_{2}:=\gamma^{m+1}|H D| \varphi(H), \quad b_{2}:=\gamma^{m+1}(2|H D|+|H A|) \varphi(H), \\
& d_{2}=e_{2}:=\gamma^{m+1}|H| \varphi(H) .
\end{aligned}
$$

Moreover, we set

$$
\begin{aligned}
& \mathcal{D}_{1}:=\min \left\{\xi_{0}, \xi_{1 a}, \xi_{1 b}, \xi_{2}\right\}, \\
& \mathcal{D}_{2}:=\min \left\{\eta_{0}, \eta_{1 a}, \eta_{1 b}, \eta_{2}\right\}
\end{aligned}
$$

with 
$\xi_{0}, \eta_{0}$ defined by equations (18), (19) where $L_{0}, a_{0}, b_{0}, c_{0}, d_{0}$ and $e_{0}$ are defined by (29)-(31),

$\xi_{1 a}, \eta_{1 a}$ defined by equations (22), (23) for $\xi_{1}, \eta_{1}$ (in (23) is $\xi_{1}$ replaced by $\xi_{1 a}$ ) where $L_{1}=L_{11}, a_{1}=a_{11}, b_{1}=b_{11}, c_{1}=c_{11}, d_{1}=d_{11}$ and $L_{11}, a_{11}, b_{11}, c_{11}, d_{11}$ are defined by the right-hand sides of (32), (33),

$\xi_{1 b}, \eta_{1 b}$ defined by equations (22), (23) for $\xi_{1}, \eta_{1}$ (in (23) is $\xi_{1}$ replaced by $\xi_{1 b}$ ) where $L_{1}=L_{12}, a_{1}=a_{12}, b_{1}=b_{12}, c_{1}=c_{12}, d_{1}=d_{12}$ and $L_{12}, a_{12}, b_{12}, c_{12}, d_{12}$ are defined by the right-hand sides of (34), (35),

$\eta_{2}, \xi_{2}$ defined by equations (26), (27) where $L_{2}, a_{2}, b_{2}, d_{2}$ and $e_{2}$ are defined by

(36), (37).

The following theorem provides us with sufficient conditions for the exponential stability and an estimate of the exponential rate of convergence of solutions.

Theorem 2 Let $\rho(A)<1, C$ be a fixed positive definite matrix and let matrix $H$ solve the corresponding Lyapunov matrix equation (6). Assume that, for a fixed $\gamma>1$, the inequalities $L_{0}>0, L_{11}>0, L_{12}>0$, and $L_{2}>0$ hold. If

$$
\begin{aligned}
& |B(k)| \leq \mathcal{D}_{1}^{*}, \quad k \geq 0, \\
& |E(k)| \leq \mathcal{D}_{2}^{*}, \quad k \geq 0,
\end{aligned}
$$

where $\mathcal{D}_{1}^{*}<\mathcal{D}_{1}, \mathcal{D}_{2}^{*}<\mathcal{D}_{2}$, then system (7) is exponentially stable and, for an arbitrary solution $x(k)$ of (7), an exponential convergence estimate

$$
|x(k)| \leq \sqrt{\varphi(H)}\|x(0)\|_{m} \gamma^{-k / 2}, \quad k \geq 0,
$$

holds.

Proof We use the Lyapunov function $V(x, k)=\gamma^{k} x^{T} H x$. Obviously,

$$
\gamma^{k} \lambda_{\min }(H)|x|^{2} \leq V(x, k) \leq \gamma^{k} \lambda_{\max }(H)|x|^{2} .
$$

For an arbitrary $\varepsilon>0$, we choose $\delta(\varepsilon):=\varepsilon / \sqrt{\varphi(H)}$. If a solution $x(k)$ of (7) satisfies $\|x(0)\|_{m}=\delta(\varepsilon)$, then, for $k=-m,-m+1, \ldots, 0$,

$$
\begin{aligned}
V(x(k), k) & \leq \gamma^{k} \lambda_{\max }(H)|x(k)|^{2} \leq \gamma^{k} \lambda_{\max }(H)\|x(0)\|_{m}^{2} \\
& =\gamma^{k} \lambda_{\max }(H) \delta^{2}(\varepsilon)=\gamma^{k} \lambda_{\max }(H) \frac{\varepsilon^{2}}{\varphi(H)} \leq \varepsilon^{2} \lambda_{\min }(H)
\end{aligned}
$$

or

$$
V(x(k), k) \leq \varepsilon^{2} \lambda_{\text {min }}(H) .
$$

We show that (42) holds for all $k \geq 1$ as well. Suppose this is not the case. Then there exists $k=k_{0} \geq 0$, such that

$$
\begin{aligned}
& V(x(k), k) \leq \varepsilon^{2} \lambda_{\min }(H), \quad k=-m,-m+1, \ldots, k_{0}, \\
& V\left(x\left(k_{0}+1\right), k_{0}+1\right)>\varepsilon^{2} \lambda_{\min }(H) \geq V\left(x\left(k_{0}\right), k_{0}\right),
\end{aligned}
$$


i.e., at $k=k_{0}+1$ for the first time, the function value $x\left(k_{0}+1\right)$ lies outside the area

$$
V^{\alpha}=\{k=-m,-m+1, \ldots: V(x(k), k) \leq \alpha\},
$$

where $\alpha=\varepsilon^{2} \lambda_{\min }(H)$. The first difference of $V(x, k)$ along the trajectories of system (7) has the form

$$
\begin{aligned}
\Delta V(x(k), k)= & \gamma^{k+1}[(A+B(k)) x(k)+(D+E(k)) x(k-m)]^{T} H \\
& \times[(A+B(k)) x(k)+(D+E(k)) x(k-m)]-\gamma^{k} x^{T}(k) H x(k) .
\end{aligned}
$$

We rewrite this expression as follows:

$$
\begin{aligned}
\Delta V(x(k), k)= & \gamma^{k+1}[A x(k)+D x(k-m)]^{T} H[A x(k)+D x(k-m)] \\
& +2 \gamma^{k+1}[B(k) x(k)+E(k) x(k-m)]^{T} H[A x(k)+D x(k-m)] \\
& +\gamma^{k+1}[B(k) x(k)+E(k) x(k-m)]^{T} H[B(k) x(k)+E(k) x(k-m)] \\
& -\gamma^{k} x^{T}(k) H x(k) .
\end{aligned}
$$

A further reorganization of the right-hand side of the last expression leads to

$$
\begin{aligned}
\Delta V(x(k), k)= & -\gamma^{k+1} x^{T}(k)\left[H-A^{T} H A\right] x(k)+2 \gamma^{k+1} x^{T}(k) A^{T} H D x(k-m) \\
& +\gamma^{k+1} x^{T}(k-m) D^{T} H D x(k-m) \\
& +2 \gamma^{k+1}\left[x^{T}(k) B^{T}(k) H A x(k)+x^{T}(k) B^{T}(k) H D x(k-m)\right. \\
& \left.+x^{T}(k-m) E^{T}(k) H A x(k)+x^{T}(k-m) E^{T}(k) H D x(k-m)\right] \\
& +\gamma^{k+1}\left[x^{T}(k) B^{T}(k) H B(k) x(k)+2 x^{T}(k) B^{T}(k) H E(k) x(k-m)\right. \\
& \left.+x^{T}(k-m) E^{T}(k) H E(k) x(k-m)\right]+\gamma^{k}(\gamma-1) x^{T} H x .
\end{aligned}
$$

Using the Lyapunov matrix equation (6) and estimating the right-hand side, we get, for $k=k_{0}$,

$$
\begin{aligned}
V(x & \left.\left(k_{0}+1\right), k_{0}+1\right)-V\left(x\left(k_{0}\right), k_{0}\right) \\
\leq & -\gamma^{k_{0}+1} \lambda_{\min }(C)\left|x\left(k_{0}\right)\right|^{2}+2 \gamma^{k_{0}+1}\left|A^{T} H D\right|\left|x\left(k_{0}\right)\right|\left|x\left(k_{0}-m\right)\right| \\
& +\gamma^{k_{0}+1}\left|D^{T} H D\right|\left|x\left(k_{0}-m\right)\right|^{2}+2 \gamma^{k_{0}+1}\left[|H A|\left|B\left(k_{0}\right)\right|\left|x\left(k_{0}\right)\right|^{2}\right. \\
& \left.+\left(|H D|\left|B\left(k_{0}\right)\right|+|H A|\left|E\left(k_{0}\right)\right|\right)\left|x\left(k_{0}\right)\right|\left|x\left(k_{0}-m\right)\right|+|H D|\left|E\left(k_{0}\right)\right|\left|x\left(k_{0}-m\right)\right|^{2}\right] \\
& +\gamma^{k_{0}+1}|H|\left[\left|B\left(k_{0}\right)\right|^{2}\left|x\left(k_{0}\right)\right|^{2}+2\left|B\left(k_{0}\right)\right|\left|E\left(k_{0}\right)\right|\left|x\left(k_{0}\right)\right|\left|x\left(k_{0}-m\right)\right|\right. \\
& \left.+\left|E\left(k_{0}\right)\right|^{2}\left|x\left(k_{0}-m\right)\right|^{2}\right]+\gamma^{k_{0}}(\gamma-1)|H|\left|x\left(k_{0}\right)\right|^{2}
\end{aligned}
$$

and, applying inequality $x\left(k_{0}\right) x\left(k_{0}-m\right) \leq\left(x^{2}\left(k_{0}\right)+x^{2}\left(k_{0}-m\right)\right) / 2$,

$$
\begin{aligned}
& V\left(x\left(k_{0}+1\right), k_{0}+1\right)-V\left(x\left(k_{0}\right), k_{0}\right) \\
& \quad \leq-\gamma^{k_{0}+1} \lambda_{\min }(C)\left|x\left(k_{0}\right)\right|^{2}
\end{aligned}
$$




$$
\begin{aligned}
& +\gamma^{k_{0}+1}\left|A^{T} H D\right|\left[\left|x\left(k_{0}\right)\right|^{2}+\left|x\left(k_{0}-m\right)\right|^{2}\right] \\
& +\gamma^{k_{0}+1}\left|D^{T} H D\right|\left|x\left(k_{0}-m\right)\right|^{2}+2 \gamma^{k_{0}+1}|H A|\left|B\left(k_{0}\right)\right|\left|x\left(k_{0}\right)\right|^{2} \\
& +\gamma^{k_{0}+1}\left(|H D|\left|B\left(k_{0}\right)\right|+|H A|\left|E\left(k_{0}\right)\right|\right)\left[\left|x\left(k_{0}\right)\right|^{2}+\left|x\left(k_{0}-m\right)\right|^{2}\right] \\
& +2 \gamma^{k_{0}+1}|H D|\left|E\left(k_{0}\right)\right|\left|x\left(k_{0}-m\right)\right|^{2} \\
& +\gamma^{k_{0}+1}|H|\left|B\left(k_{0}\right)\right|^{2}\left|x\left(k_{0}\right)\right|^{2}+\gamma^{k_{0}+1}|H|\left|B\left(k_{0}\right)\right|\left|E\left(k_{0}\right)\right|\left[\left|x\left(k_{0}\right)\right|^{2}+\left|x\left(k_{0}-m\right)\right|^{2}\right] \\
& +\gamma^{k_{0}+1}|H|\left|E\left(k_{0}\right)\right|^{2}\left|x\left(k_{0}-m\right)\right|^{2}+\gamma^{k_{0}}(\gamma-1)|H|\left|x\left(k_{0}\right)\right|^{2} .
\end{aligned}
$$

Since the chain of inequalities

$$
\begin{aligned}
\gamma^{k} \lambda_{\min }(H)|x(k)|^{2} & \leq V(x(k), k) \leq \alpha=\varepsilon^{2} \lambda_{\min }(H) \\
& <V\left(x\left(k_{0}+1\right), k_{0}+1\right) \\
& \leq \gamma^{k_{0}+1} \lambda_{\max }(H)\left|x\left(k_{0}+1\right)\right|^{2}
\end{aligned}
$$

holds for $k=-m,-m+1, \ldots, k_{0}$, we deduce

$$
|x(k)|<\sqrt{\varphi(H)}\left|x\left(k_{0}+1\right)\right| \gamma^{\left(k_{0}+1-k\right) / 2}, \quad k=-m,-m+1, \ldots, k_{0} .
$$

Applying (44) in the last expression of the estimate of the first difference of the Lyapunov function $V(x(k), k)$ at $k=k_{0}$ to terms with the argument $k_{0}-m$, i.e., putting $k=k_{0}-m$ in (44), we get

$$
\begin{aligned}
V(x( & \left.\left.k_{0}+1\right), k_{0}+1\right)-V\left(x\left(k_{0}\right), k_{0}\right) \\
\leq & -\gamma^{k_{0}+1}\left(\lambda_{\min }(C)-\left|A^{T} H D\right|\right)\left|x\left(k_{0}\right)\right|^{2} \\
& +\gamma^{\left(k_{0}+1\right)+(m+1)}\left[\left|A^{T} H D\right|+\left|D^{T} H D\right|\right] \varphi(H)\left|x\left(k_{0}+1\right)\right|^{2} \\
& +\gamma^{k_{0}+1}\left[(2|H A|+|H D|)\left|B\left(k_{0}\right)\right|+|H A|\left|E\left(k_{0}\right)\right|\right]\left|x\left(k_{0}\right)\right|^{2} \\
& +\gamma^{\left(k_{0}+1\right)+(m+1)}\left[(2|H D|+|H A|)\left|E\left(k_{0}\right)\right|+|H D|\left|B\left(k_{0}\right)\right|\right] \varphi(H)\left|x\left(k_{0}+1\right)\right|^{2} \\
& +\gamma^{k_{0}+1}|H|\left|B\left(k_{0}\right)\right|\left[\left|B\left(k_{0}\right)\right|+\left|E\left(k_{0}\right)\right|\right]\left|x\left(k_{0}\right)\right|^{2} \\
& +\gamma^{\left(k_{0}+1\right)+(m+1)}|H|\left|E\left(k_{0}\right)\right|\left[\left|B\left(k_{0}\right)\right|+\left|E\left(k_{0}\right)\right|\right] \varphi(H)\left|x\left(k_{0}+1\right)\right|^{2} \\
& +\gamma^{k_{0}}(\gamma-1)|H|\left|x\left(k_{0}\right)\right|^{2} .
\end{aligned}
$$

We can rewrite this inequality, adding up the multiples of $\left|x\left(k_{0}\right)\right|^{2}$ and of $\left|x\left(k_{0}+1\right)\right|^{2}$, as

$$
\begin{aligned}
V(x( & \left.\left.k_{0}+1\right), k_{0}+1\right)-V\left(x\left(k_{0}\right), k_{0}\right) \\
\leq & -\gamma^{k_{0}+1}\left(\lambda_{\min }(C)-\left|A^{T} H D\right|-\left((2|H A|+|H D|)\left|B\left(k_{0}\right)\right|+|H A|\left|E\left(k_{0}\right)\right|\right)\right. \\
& \left.-|H|\left|B\left(k_{0}\right)\right|\left(\left|B\left(k_{0}\right)\right|+\left|E\left(k_{0}\right)\right|\right)-(1-1 / \gamma)|H|\right)\left|x\left(k_{0}\right)\right|^{2} \\
& +\gamma^{\left(k_{0}+1\right)+(m+1)}\left(\left|A^{T} H D\right|+\left|D^{T} H D\right|+(2|H D|+|H A|)\left|E\left(k_{0}\right)\right|+|H D|\left|B\left(k_{0}\right)\right|\right. \\
& \left.+|H|\left|E\left(k_{0}\right)\right|\left(\left|B\left(k_{0}\right)\right|+\left|E\left(k_{0}\right)\right|\right)\right) \varphi(H)\left|x\left(k_{0}+1\right)\right|^{2} .
\end{aligned}
$$


We derive sufficient conditions for the positivity of the first coefficient in the round brackets in (45), i.e., for

$$
\begin{gathered}
\lambda_{\min }(C)-\left|A^{T} H D\right|-\left((2|H A|+|H D|)\left|B\left(k_{0}\right)\right|+|H A|\left|E\left(k_{0}\right)\right|\right) \\
-|H|\left|B\left(k_{0}\right)\right|\left(\left|B\left(k_{0}\right)\right|+\left|E\left(k_{0}\right)\right|\right)-(1-1 / \gamma)|H|>0 .
\end{gathered}
$$

We apply Lemma 4 with $L_{1}:=L_{11}, a_{1}:=a_{11}, b_{1}:=b_{11}, c_{1}:=c_{11}, d_{1}:=d_{11}$ where $L_{11}, a_{11}, b_{11}, c_{11}$, and $d_{11}$ are defined by (32), (33) (obviously $c_{11}=d_{11}>0$ ). It is sufficient to satisfy inequality (21), i.e.,

$$
\begin{aligned}
& \left|B\left(k_{0}\right)\right|<\xi, \quad \text { where we set } \xi=\mathcal{D}_{1}^{*}, \\
& \left|E\left(k_{0}\right)\right|<\eta, \quad \text { where we set } \eta=\mathcal{D}_{2}^{*} .
\end{aligned}
$$

Both inequalities are valid due to assumptions (38), (39).

Therefore, we can continue to estimate the first difference of the Lyapunov function. Using (41), from (45), we get

$$
\begin{aligned}
& V\left(x\left(k_{0}+1\right), k_{0}+1\right)-V\left(x\left(k_{0}\right), k_{0}\right) \\
& \leq- \gamma\left(\lambda_{\min }(C)-\left|A^{T} H D\right|-\left[(2|H A|+|H D|)\left|B\left(k_{0}\right)\right|+|H A|\left|E\left(k_{0}\right)\right|\right]\right. \\
&\left.-|H|\left|B\left(k_{0}\right)\right|\left(\left|B\left(k_{0}\right)\right|+\left|E\left(k_{0}\right)\right|\right)-(1-1 / \gamma)|H|\right) \frac{V\left(x\left(k_{0}\right), k_{0}\right)}{\lambda_{\max }(H)} \\
&+\gamma^{m+1}\left(\left|A^{T} H D\right|+\left|D^{T} H D\right|+(2|H D|+|H A|)\left|E\left(k_{0}\right)\right|+|H D|\left|B\left(k_{0}\right)\right|\right. \\
&\left.+|H|\left|E\left(k_{0}\right)\right|\left(\left|B\left(k_{0}\right)\right|+\left|E\left(k_{0}\right)\right|\right)\right) \frac{\varphi(H) V\left(x\left(k_{0}+1\right), k_{0}+1\right)}{\lambda_{\min }(H)} .
\end{aligned}
$$

The resulting inequality can be rewritten in the form

$$
\begin{aligned}
{[1-} & \gamma^{m+1}\left(\left|A^{T} H D\right|+\left|D^{T} H D\right|+(2|H D|+|H A|)\left|E\left(k_{0}\right)\right|+|H D|\left|B\left(k_{0}\right)\right|\right. \\
& \left.\left.+|H|\left|E\left(k_{0}\right)\right|\left(\left|B\left(k_{0}\right)\right|+\left|E\left(k_{0}\right)\right|\right)\right) \frac{\varphi(H)}{\lambda_{\min }(H)}\right] V\left(x\left(k_{0}+1\right), k_{0}+1\right) \\
\leq & {\left[1-\gamma\left(\lambda_{\min }(C)-\left|A^{T} H D\right|-\left[(2|H A|+|H D|)\left|B\left(k_{0}\right)\right|+|H A|\left|E\left(k_{0}\right)\right|\right]\right.\right.} \\
& \left.\left.\quad-|H|\left|E\left(k_{0}\right)\right|\left[\left|B\left(k_{0}\right)\right|+\left|E\left(k_{0}\right)\right|\right]-(1-1 / \gamma)|H|\right) \frac{1}{\lambda_{\max }(H)}\right] V\left(x\left(k_{0}\right), k_{0}\right) .
\end{aligned}
$$

We derive sufficient conditions for the positivity of coefficients in the big square brackets, i.e., for

$$
\begin{aligned}
\Theta_{1}\left(k_{0}\right):= & \lambda_{\min }(H)-\gamma^{m+1}\left(\left|A^{T} H D\right|+\left|D^{T} H D\right|+(2|H D|+|H A|)\left|E\left(k_{0}\right)\right|\right. \\
& \left.+|H D|\left|B\left(k_{0}\right)\right|+|H|\left|E\left(k_{0}\right)\right|\left(\left|B\left(k_{0}\right)\right|+\left|E\left(k_{0}\right)\right|\right)\right) \varphi(H)>0
\end{aligned}
$$

and

$$
\begin{aligned}
\Theta_{2}\left(k_{0}\right):= & \lambda_{\max }(H)-\gamma\left(\lambda_{\min }(C)-\left|A^{T} H D\right|-\left((2|H A|+|H D|)\left|B\left(k_{0}\right)\right|+|H A|\left|E\left(k_{0}\right)\right|\right)\right. \\
& \left.-|H|\left|B\left(k_{0}\right)\right|\left(\left|B\left(k_{0}\right)\right|+\left|E\left(k_{0}\right)\right|\right)-(1-1 / \gamma)|H|\right)>0 .
\end{aligned}
$$


Consider inequality (46). We apply Lemma 5 with $L_{2}, a_{2}, b_{2}, d_{2}$, and $e_{2}$ defined by (36),

(37) (obviously $d_{2}=e_{2}>0$ ). It is sufficient to satisfy the inequalities (25), i.e.,

$$
\begin{aligned}
& \left|B\left(k_{0}\right)\right|<\xi, \quad \text { where we set } \xi=\mathcal{D}_{1}^{*}, \\
& \left|E\left(k_{0}\right)\right|<\eta, \quad \text { where we set } \eta=\mathcal{D}_{2}^{*} .
\end{aligned}
$$

Both inequalities are valid due to assumptions (38), (39).

Consider inequality (47). We apply Lemma 4 with $L_{1}:=L_{12}, a_{1}:=a_{12}, b_{1}:=b_{12}, c_{1}:=c_{12}$, $d_{1}:=d_{12}$ where $L_{12}, a_{12}, b_{12}, c_{12}$, and $d_{12}$ are defined by (34), (35) (obviously $c_{12}=d_{12}>0$ ). It is sufficient to satisfy inequality (21), i.e.,

$$
\begin{aligned}
& \left|B\left(k_{0}\right)\right|<\xi, \quad \text { where we set } \xi=\mathcal{D}_{1}^{*}, \\
& \left|E\left(k_{0}\right)\right|<\eta, \quad \text { where we set } \eta=\mathcal{D}_{2}^{*} .
\end{aligned}
$$

Both inequalities are valid due to assumptions (38), (39).

Then for an estimate of the Lyapunov function we get the inequality

$$
V\left(x\left(k_{0}+1\right), k_{0}+1\right) \leq \Theta\left(k_{0}\right) V\left(x\left(k_{0}\right), k_{0}\right),
$$

where

$$
\Theta\left(k_{0}\right):=\frac{\Theta_{2}\left(k_{0}\right)}{\Theta_{1}\left(k_{0}\right) \varphi(H)} .
$$

We show that

$$
0<\Theta\left(k_{0}\right)<1 .
$$

The left-hand side of (49) holds due to the above arguments. The inequality

$$
\Theta_{1}\left(k_{0}\right) \varphi(H)>\Theta_{2}\left(k_{0}\right)
$$

is a sufficient condition for the right-hand side of (49) to be valid, i.e. (after a minor rearrangement),

$$
\begin{aligned}
\lambda_{\min }(C) & -\left|A^{T} H D\right| \\
& -\left((2|H A|+|H D|)\left|B\left(k_{0}\right)\right|+|H A|\left|E\left(k_{0}\right)\right|\right) \\
& -|H|\left|B\left(k_{0}\right)\right|\left(\left|B\left(k_{0}\right)\right|+\left|E\left(k_{0}\right)\right|\right)-(1-1 / \gamma)|H| \\
> & \gamma^{m}\left(\left|A^{T} H D\right|+\left|D^{T} H D\right|+(2|H D|+|H A|)\left|E\left(k_{0}\right)\right|+|H D|\left|B\left(k_{0}\right)\right|\right. \\
& \left.+|H|\left|E\left(k_{0}\right)\right|\left[\left|B\left(k_{0}\right)\right|+\left|E\left(k_{0}\right)\right|\right]\right) \varphi^{2}(H)
\end{aligned}
$$

or

$$
\begin{aligned}
& \lambda_{\min }(C)-\left|A^{T} H D\right|\left(1+\gamma^{m} \varphi^{2}(H)\right)-\gamma^{m}\left|D^{T} H D\right| \varphi^{2}(H)-(1-1 / \gamma)|H| \\
& \quad-\left(2|H A|+|H D|\left(1+\gamma^{m} \varphi^{2}(H)\right)\right)\left|B\left(k_{0}\right)\right|
\end{aligned}
$$




$$
\begin{aligned}
& -\left(|H A|+(2|H D|+|H A|) \gamma^{m} \varphi^{2}(H)\right)\left|E\left(k_{0}\right)\right|-|H|\left|B\left(k_{0}\right)\right|^{2} \\
& -|H|\left(1+\gamma^{m} \varphi^{2}(H)\right)\left|B\left(k_{0}\right)\right|\left|E\left(k_{0}\right)\right|-|H| \gamma^{m} \varphi^{2}(H)\left|E\left(k_{0}\right)\right|^{2}>0 .
\end{aligned}
$$

As follows from (29), (30), and (31), inequality (50) can be rewritten as

$$
L_{0}-a_{0}\left|B\left(k_{0}\right)\right|-b_{0}\left|E\left(k_{0}\right)\right|-c_{0}\left|B\left(k_{0}\right)\right|^{2}-d_{0}\left|B\left(k_{0}\right)\right|\left|E\left(k_{0}\right)\right|-e_{0}\left|E\left(k_{0}\right)\right|^{2}>0 .
$$

We apply Lemma 3 with $L_{0}, a_{0}, b_{0}, c_{0}, d_{0}$, and $e_{0}$ defined by (29)-(31) (obviously $c_{0}>0$, $\left.e_{0}>0\right)$. It is sufficient to satisfy inequality (17), i.e.,

$$
\begin{aligned}
& \left|B\left(k_{0}\right)\right|<\xi, \quad \text { where we set } \xi=\mathcal{D}_{1}^{*}, \\
& \left|E\left(k_{0}\right)\right|<\eta, \quad \text { where we set } \eta=\mathcal{D}_{2}^{*} .
\end{aligned}
$$

Both inequalities are valid due to assumptions (38), (39). Consequently, (49) holds and, from (43), we get

$$
V\left(x\left(k_{0}\right), k_{0}\right)<V\left(x\left(k_{0}+1\right), k_{0}+1\right)<V\left(x\left(k_{0}\right), k_{0}\right) .
$$

Therefore, assumption (43) is false and (42) holds for every $k \geq-m$. From (41) and (42) we get

$$
\gamma^{k} \lambda_{\min }(H)|x|^{2} \leq V(x, k) \leq \varepsilon^{2} \lambda_{\min }(H)=\delta^{2}(\varepsilon) \lambda_{\max }(H)=\|x(0)\|_{m}^{2} \lambda_{\max }(H)
$$

where $k \geq 0$, and

$$
|x(k)| \leq \sqrt{\varphi(H)}\|x(0)\|_{m} \gamma^{-k / 2}, \quad k \geq 0,
$$

i.e., equation (40) holds.

Example 2 Let $n=2, m=10$,

$$
A=\left(\begin{array}{ll}
0.7 & 0.1 \\
0.1 & 0.7
\end{array}\right)
$$

and, for simplicity, $D$ be a $2 \times 2$ null matrix specified in system (7). Let

$$
C=\left(\begin{array}{cc}
0.5 & -0.14 \\
-0.14 & 0.5
\end{array}\right) \text {. }
$$

The matrix

$$
H=\left(\begin{array}{ll}
1 & 0 \\
0 & 1
\end{array}\right)
$$

solves the matrix Lyapunov equation (6). As in Example 2, we get $\lambda_{\min }(C)=0.36, \lambda_{\min }(H)=$ $\lambda_{\max }(H)=\varphi(H)=1,|H|=1$ and $\left|A^{T} H\right|=0.8$. Moreover, let $\gamma=1.01$. Then

$$
L_{0}=\lambda_{\min }(C)-(1-1 / \gamma)|H| \doteq 0.3501,
$$




$$
\begin{aligned}
& a_{0}=2|H A|=1.6, \quad b_{0}=|H A|+|H A| \gamma \varphi^{2}(H)=1.608, \\
& c_{0}=|H|=1, \quad d_{0}=\left(1+\gamma \varphi^{2}(H)\right)|H|=2.01, \quad e_{0}=\gamma|H| \varphi^{2}(H)=1.01, \\
& L_{11}=L_{0} \doteq 0.3501, \\
& a_{11}=a_{0}=1.6, \quad b_{11}=|H A|=0.8, \quad c_{11}=d_{11}=|H|=1, \\
& L_{12}=\lambda_{\max }(H)-\gamma \lambda_{\min }(C)-(\gamma-1)|H| \doteq 0.6264, \\
& a_{12}=2 \gamma|H A|=1.616, \quad b_{12}=\gamma|H A|=0.808, \quad c_{12}=d_{12}=\gamma|H|=1.01, \\
& L_{2}=\lambda_{\min }(H)=1, \\
& a_{2}=0, \quad b_{2}=\gamma^{m+1}|H A| \varphi(H) \doteq 0.8925, \quad d_{2}=e_{2}=\gamma^{m+1}|H| \varphi(H) \doteq 1.1157,
\end{aligned}
$$

and

$$
\begin{array}{ll}
\xi_{0} \doteq 0.1028, & \eta_{0} \doteq 0.0918 \\
\xi_{1 a} \doteq 0.1028, & \eta_{1 a} \doteq 0.1939, \\
\xi_{1 b} \doteq 0.1747, & \eta_{1 a} \doteq 0.3181, \\
\xi_{2} \doteq 1.1797, & \eta_{2} \doteq 0.3799 .
\end{array}
$$

Let

$$
0<\mathcal{D}_{1}^{*}<\mathcal{D}_{1}=\min \left\{\xi_{0}, \xi_{1 a}, \xi_{1 b}, \xi_{2}\right\}=\xi_{0} \doteq 0.1028
$$

and

$$
0<\mathcal{D}_{2}^{*}<\mathcal{D}_{2}=\min \left\{\eta_{0}, \eta_{1 a}, \eta_{1 b}, \eta_{2}\right\}=\eta_{0} \doteq 0.0918
$$

If $|B(k)| \leq \mathcal{D}_{1}^{*},|E(k)| \leq \mathcal{D}_{2}^{*}, k=0,1, \ldots$, then system (7) is exponentially stable. From (40), we get

$$
|x(k)| \leq \sqrt{\varphi(H)}\|x(0)\|_{m} \gamma^{-k / 2}=(1.01)^{-k / 2} \cdot\|x(0)\|_{10}, \quad k \geq 0 .
$$

\section{Concluding remarks}

The paper investigates the exponential stability assuming, without loss of generality, that the initial point is always defined for the value of the independent variable $k$ being equal to 0 in the case of the non-delayed equation (2), and that the initial functions in the case of the delayed systems (7) are always defined for $k=-m,-m+1, \ldots, 0$. Obviously, our investigation can easily be modified for an arbitrary integer value of independent variable $k=k_{0}$ in the case of the non-delayed equation (2) or if the initial functions, in the case of the delayed systems (7) are defined for the values of $k=k_{0}-m, k_{0}-m+1, \ldots, k_{0}$. To this end, some minor changes in Definitions 1 and 2 are necessary. We omit the details as well as the relevant reformulation of the results.

Carefully tracing the proof of Theorem 1 we note that system (2) will be exponentially stable if condition (11) is replaced by a more general assumption:

$$
\limsup _{k \rightarrow \infty}|B(k)|<\mathcal{D}^{*} .
$$


Nevertheless, in such a case, inequality (12) is, in general, not preserved because the constant $N:=\sqrt{\varphi(H)}$ in (12) (see Definition 1) can be different. Similarly, the exponential stability of system (7) will be preserved if inequalities (38), (39) in Theorem 2 are replaced by

$$
\begin{gathered}
\limsup _{k \rightarrow \infty}|B(k)|<\mathcal{D}_{1}^{*}, \\
\limsup _{k \rightarrow \infty}|E(k)|<\mathcal{D}_{2}^{*} .
\end{gathered}
$$

In such a case, inequality (40) will be valid if, in general, the constant $N:=\sqrt{\varphi(H)}$ in (9) (see Definition 2) is different. An open problem arises if we discuss inequalities (53) and (54), (55) concerning their further improvement. Particularly, is it possible to replace (9) by the weaker condition

$$
\limsup _{k \rightarrow \infty} \rho(B(k))<\mathcal{D}^{*}
$$

and (54), (55) by the weaker conditions

$$
\begin{aligned}
& \limsup _{k \rightarrow \infty} \rho(B(k))<\mathcal{D}_{1}^{*}, \\
& \limsup _{k \rightarrow \infty} \rho(E(k))<\mathcal{D}_{2}^{*},
\end{aligned}
$$

where $\rho$ is the spectral radius of a matrix?

The admissible values of the parameter $\gamma$ used in the proof of Theorem 2 are $\gamma>1$. Setting $\gamma=1$ and tracing all steps of the proof, we conclude that the assertion of exponential stability is not true. It can be seen, e.g., from (52), that, in such a case, only the stability is proved since, for $\|x(0)\|_{m}=\delta(\varepsilon)$ and $k \geq 0$, from (52) we get

$$
|x(k)| \leq \sqrt{\varphi(H)}\|x(0)\|_{m} \gamma^{-k / 2}=\sqrt{\varphi(H)}\|x(0)\|_{m}=\sqrt{\varphi(H)} \delta(\varepsilon)=\sqrt{\varphi(H)} \varepsilon / \sqrt{\varphi(H)}=\varepsilon .
$$

Let $K$ be a set of consecutive integers such that $k \geq r$ for every $k \in K$ ( $r$ is an integer). In [7] an exponential estimate of solutions of linearly perturbed linear systems

$$
y(k+1)=\left[A_{1}(k)+B_{1}(k)\right] y(k), \quad k \in K,
$$

is studied assuming that matrices $A_{1}(k)$ and $B_{1}(k)$ are defined on $K,\left\|B_{1}(k)\right\| \leq \beta, k \in K$, $\|\cdot\|$ is a norm, and all solutions of the non-perturbed linear system

$$
x(k+1)=A_{1}(k) x(k), \quad k \in K,
$$

satisfy the estimate

$$
\|x(k)\| \leq q\|x(r)\| \alpha^{k-r}, \quad k \in K,
$$

for some positive constants $q$ and $\alpha$. 
Then, by Theorem 2, part (i) in [7] any (forward) solution $y(k)$ of (56) satisfies

$$
\|y(k)\| \leq q\|y(r)\|(\alpha+\beta q)^{k-r}=\frac{q}{(\alpha+\beta q)^{r}}\|y(r)\|\left[(\alpha+\beta q)^{2}\right]^{k / 2}, \quad k \in K .
$$

To compare our results with those described above we assume $\alpha<1$ in (58) (in the case of constant matrix $A_{1}$, this inequality is equivalent to the assumption $\rho(A)<1$ in Theorem 1 ).

Compare estimate (59) with estimation (12) in Theorem 1. The first one gives a qualitative result (since an estimate for $q$ is not given and, therefore, it is not clear how small $\beta$ should be to guarantee the inequality $\alpha+\beta q<1$ and we cannot use (59) for numerical computations). The constants

$$
\sqrt{\varphi(H)} \text { and } 1-\frac{\xi}{\lambda_{\max }(H)}
$$

in (12) play the roles of the constants $q(\alpha+\beta q)^{-r}$ and $(\alpha+\beta q)^{2}$ in (59). We conclude that inequality (12) gives, together with qualitative information, a quantitative result as well and can be used for computational purposes.

Let $B(k)=\Theta$ for every $k=1,2, \ldots$, where $\Theta$ is a $n \times n$ null matrix. Then we can set $\mathcal{D}:=0$ and $\xi=\lambda_{\min }(C)$. In such a case, Theorem 1 becomes the following theorem.

Theorem 3 Let $\rho(A)<1$. Let $C$ be a fixed positive definite matrix, and let matrix $H$ solve matrix equation (6). Then system (1) is exponentially stable and, for any of its solutions $x=x(k)$, the inequality

$$
|x(k)| \leq\left[1-\frac{\lambda_{\min }(C)}{\lambda_{\max }(H)}\right]^{k / 2} \sqrt{\varphi(H)}|x(0)|, \quad k \geq 0,
$$

holds where

$$
0<1-\frac{\lambda_{\min }(C)}{\lambda_{\max }(H)}<1
$$

Comparing inequality (60) with (58) (and assuming, for simplicity, that $A_{1}(k)$ in (57) is a constant matrix) we can explain the differences between both inequalities in a way similar to the one we used above.

Let us discuss the relationship between Theorem 1 and Theorem 2 . It is obvious that they are independent since, in the case of system (7), we assume $m \geq 1$. Moreover, setting $D=\Theta$ and $E(k) \equiv \Theta$ in system (7), where $\Theta$ is the zero matrix, in order to turn it into system (1), does not turn the conclusion of Theorem 2 to the conclusion of Theorem 1. A direct comparison leads to a conclusion that the latter is sharper. The reason for this is that the auxiliary inequalities (Lemmas 3, 4, and 5), used in the proof of Theorem 2, are rather imprecise and cannot imply the same result.

The authors have recently published papers $[19,20]$ on a similar topic where further related results can be found, including their application to scalar equations and their comparison with known results. In [21] the boundedness character of positive solutions is studied. Let us refer as well to [22] where some exact stability results are derived. Although our results cannot give such exact results, the advantage of our approach is that the estimates of the norms of the solutions are given by inequalities. Such estimates are not derived in [22]. 


\section{Competing interests}

The authors declare that they have no competing interests.

\section{Authors' contributions}

All authors contributed equally to the writing of this paper. All authors read and approved the final manuscript.

\section{Author details}

${ }^{1}$ Brno University of Technology, Brno, Czech Republic. ${ }^{2}$ Kiev State University, Kiev, Ukraine.

\section{Acknowledgements}

The first author has been supported by the project No. LO1408, AdMaS UP - Advanced Materials, Structures and Technologies (supported by Ministry of Education, Youth and Sports of the Czech Republic under the National Sustainability Programme I). The second author has been supported by the project RP9051800400 (3.5 Support of International Mobility of Academic Staff, Faculty of Civil Engineering, Brno University of Technology) as a part of developing programme guaranteed by Ministry of Education, Youth and Sports of the (zech Republic). The third author has been supported by the Grant FEKT-S-14-2200 of Faculty of Electrical Engineering and Communication, Brno University of Technology.

Received: 30 September 2015 Accepted: 23 December 2015 Published online: 04 January 2016

\section{References}

1. Agarwal, RP: Difference Equations and Inequalities: Theory, Methods and Applications, 2nd edn. Monographs and Textbooks in Pure and Applied Mathematics. Dekker, New York (2000)

2. Agarwal, RP, Wong, PJY: Advanced Topics in Difference Equations. Kluwer Academic, Dordrecht (1997)

3. Aulbach, B: Continuous and Discrete Dynamics near Manifolds of Equilibria. Lecture Notes in Mathematics, vol. 1058. Springer, Berlin (1984)

4. Elaydi, SN: An Introduction to Difference Equations. Undergraduate Texts in Mathematics. Springer, Berlin (2005)

5. Kelly, WG, Peterson, AC: Difference Equations. Academic Press, San Diego (2001)

6. Laksmikantham, V, Trigiante, D: Theory of Difference Equations: Numerical Methods and Applications, 2nd edn. Dekker, New York (2002)

7. Aulbach, B: On linearly perturbed linear system. J. Math. Anal. Appl. 112, 317-327 (1985)

8. Jones, WB, Thorn, WJ: Continued Fractions: Analytic Theory and Applications. Addison-Wesley, Reading (1980)

9. Marden, M: Geometry of Polynomials. Am. Math. Soc., Providence (1966)

10. Haritonov, VL: The asymptotic stability of the equilibrium state of a family of systems of linear differential equations. Differ. Uravn. 14, 2086-2088 (1978) (in Russian) (English translation: Differ. Equ. 14, 1483-1485 (1979))

11. Čermák, J, Jánský, J: Stability switches in linear delay difference equations. Appl. Math. Comput. 243, 755-766 (2014)

12. Čermák, J, Jánský, J, Kundrát, P: On necessary and sufficient conditions for the asymptotic stability of higher order linear difference equations. J. Differ. Equ. Appl. 18, 1781-1800 (2012)

13. Čermák, J, Tomášek, P: On delay-dependent stability conditions for a three-term linear difference equation. Funkc. Ekvacioj 57, 91-106 (2014)

14. Kaslik, E: Stability results for a class of difference systems with delay. Adv. Differ. Equ. 2009, Article ID 938492 (2009)

15. Kipnis, MM, Nigmatullin, RM: Stability of the trinomial linear difference equations with two delays. Autom. Remote Control 65(11), 1710-1723 (2004)

16. Kuruklis, SA: The asymptotic stability of $x_{n+1}-a x_{n}+b x_{n-k}=0$. J. Math. Anal. Appl. 188, 719-731 (1994)

17. Levin, SA, May, R: A note on difference delay equations. Theor. Popul. Biol. 9, 178-187 (1976)

18. Bellman, R: Introduction to Matrix Analysis. McGraw-Hill, New York (1960) (Russian translation: Nauka, Moscow (1976))

19. Diblík, J, Khusainov, DY, Baštinec, J, Sirenko, AS: Stability and exponential stability of linear discrete systems with constant coefficients and single delay. Appl. Math. Comput. 269, 9-16 (2015)

20. Diblík, J, Khusainov, DY, Baštinec, J, Sirenko, AS: Exponential stability of linear discrete systems with constant coefficients and single delay. Appl. Math. Lett. 51, 68-73 (2016)

21. Stević, S: Boundedness character of the recursive sequence $x_{n}=\alpha+\prod_{j=1}^{k} x_{n-j}^{a_{j}}$. Appl. Math. Lett. 50, 83-90 (2015)

22. Kipnis, M, Komissarova, D: Stability of a delay difference system. Adv. Differ. Equ. 2006, Article ID 31409 (2006)

\section{Submit your manuscript to a SpringerOpen ${ }^{\circ}$ journal and benefit from:}

- Convenient online submission

Rigorous peer review

- Immediate publication on acceptance

- Open access: articles freely available online

- High visibility within the field

- Retaining the copyright to your article 